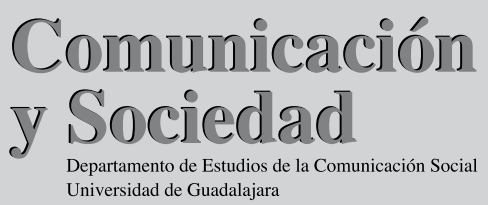

\title{
La recepción de la imagen de las mujeres en los medios: una aproximación cualitativa
}

\author{
NÚRIA GARCÍA MUÑOZ Y LUISA MARTÍNEZ GARCÍA ${ }^{1}$
}

Este artículo tiene como objetivo analizar los resultados de una investigación sobre el consumo femenino de los estereotipos de género presentes en los medios de comunicación. Nos interesa conocer cuáles son los estereotipos de género que las mujeres valoran como discriminatorias y cuáles consideran como prácticas positivas respecto a la imagen de la mujer en la sociedad. Pretendemos generar pautas de conocimiento en tres vertientes: en la percepción y consideración de la ciudadanía frente a los contenidos mediáticos, comprensión de la participación social referente a dichos contenidos, y la valoración, efectividad y otras acciones que pueden ayudar a mejorar la representación de las mujeres en los medios de comunicación.

PALABRAS ClAVE: estudios de género, audiencias, recepción, estereotipos de género, recepción.
This article aims to analyse the results of an investigation into the use of female gender stereotypes present in the media. We are interested in knowing what the gender stereotypes that women are valued as discriminatory and which regarded as positive practices regarding the image of women in society. We intend to generate patterns of knowledge in three areas: in the perception and consideration of citizenship in front of the media contents, understand the social participation about media content and the valuation strategy, effectiveness and other actions that can help improve the representation of women in the media.

KEY WORDS: gender studies, audiences, advertising, gender stereotypes, reception.

1 Facultat de Ciències de la Comunicació de la Universitat Autònoma de Barcelona.

Correos electrónicos: nuria.garcia@uab.cat / luisa.martinez@uab.cat 


\section{INTRODUCCIÓN}

Este artículo trata la percepción de las mujeres sobre los estereotipos representados en la publicidad y en los medios de comunicación ${ }^{2}$. El estudio surge como resultado de la observación de la representación femenina en los medios de comunicación. Es una investigación que se enfoca, por tanto, en la recepción, en el consumo femenino de los medios. El objetivo principal es analizar cómo las mujeres consumen las representaciones mediáticas de su mismo género. Una de las premisas que rigen este texto es la evidente relación entre el consumo de los productos mediáticos (ficción televisiva, prensa, radio, internet, publicidad, etc.) y los procesos de identificación, reconocimiento, rechazo y/o indiferencia, que la audiencia experimenta al apropiarse de los contenidos. El objetivo fundamental del artículo es exponer los resultados del análisis del consumo de los estereotipos de género, específicamente del femenino, construidos por la publicidad y otros contenidos de los medios.

\section{PRECISIONES TEÓRICAS}

En las últimas cuatro décadas los medios han sido objeto de numerosas investigaciones centradas en el estudio de los estereotipos de género. Buena parte del conocimiento se debe a las aportaciones llegadas desde disciplinas y ámbitos diversos, como la psicología, la sociología, la antropología, la lingüística, la semiótica y la comunicación. La mayoría de ellas expresan la reiterada presencia de estereotipos tradicionales y la escasa práctica de representaciones no sexistas. Dicho fenómeno ha sido validado desde propuestas y corrientes diversas, entre las que destacan estudios provenientes de las teorías feministas (Woodward 1997), desde estudios pragmáticos (Kovacs, 1972) o desde los análisis

2 El tema es el eje central de una investigación más amplia, desarrollada desde el 2005 por las autoras del artículo, financiada por el Institut Català de les Dones de la Generalitat de Catalunya y desarrollada en el GRISS (Grup de Recerca en Imatge, Só i Síntesi) del Departamento de Comunicació Audiovisual i Publicitat de la UAB (Universitat Autònoma de Barcelona). Los grupos de discusión de 2005 fueron realizados por Leonor Balbuena. 
críticos (Courtney y Whipple, 1983). Ya en la década de los sesenta se habían publicado 150 estudios sobre roles sexuales en la publicación americana "Psychological abstracts" (Ruble y Ruble, 1982) y Friedan, ya en 1963, denunció la discriminación de las mujeres en los medios de comunicación en la obra Femenine mystique.

Uno de los ejes centrales de numerosas investigaciones es y continúa siendo el estudio sobre publicidad y género a partir del análisis de contenido de las producciones de los medios (Furnham y otros, 2000; Hall, 1994; Massé y Rosenblum, 1988; Belkaoui y Belkaoui, 1980; Pingree et al., 1976; Wagner y Baños, 1973). El estudio de la producción de las industrias culturales enmarca una buena parte la investigación sobre género y medios de comunicación (Wood, 1994; Zoonen 1995; y Ceulemans y Fauconnier, 1980). Merece la pena destacar la obra de Goffman (1976), a partir de un trabajo interdisciplinar entre la semiótica y la sociología, sobre la escenificación de la imagen de la mujer en la publicidad.

A la preocupación científica sobre género y comunicación se une también la institucional. En la década de 1980, algunos organismos internacionales se ocupan por hacer una reflexión sobre la imagen de la mujer en los medios de comunicación. La UNESCO promovió, en tal década, importantes obras como las de Celeulamans y Facuconnier, (1980) y Gallagher (1981 y 1986). En este decenio, la mayoría de los estudios sobre género y medios se enfocaban al análisis de los estereotipos de género como elemento principal de las investigaciones. Los resultados radiografiaban las representaciones sexistas de los contenidos de los medios.

El estudio sobre el análisis de las personas y características de los productores de información de los medios se extiende básicamente en los años noventa. Destacan en ese momento las aportaciones de Van Zoonen (1994). También en los noventa encontramos trabajos que revelan un cierto descenso en las representaciones sexistas de los medios, principalmente en la imagen de las mujeres como elementos decorativos y en las representaciones ocupacionales (Klasse, Jasper y Schwartz 1993; Ferguson, Kreshel y Tinkham, 1990). Este fenómeno no impide la creación continuada de producciones de imágenes sexistas.

Desde los estudios culturales, también en la década de 1990, se propone una revisión de los contenidos de los medios dirigidos a las mu- 
jeres a partir del análisis crítico de los postulados de la construcción de significados basada en concepciones patriarcales (Curran et al., 1998). En esta misma obra, Walkerdine (1998) profundiza sobre la necesidad de analizar la imagen erotizada de las niñas en los medios desde un enfoque psicoanalítico y McRobbie (1998) analiza las representaciones sexuales de los adolescentes a partir de una reflexión crítica de las producciones de revistas. A finales del XX y a principios del XXI aumenta la preocupación científica por el conocimiento de los procesos comunicativos desde la perspectiva de género. Si bien es cierto que los estudios de recepción a partir de los años ochenta demostraban ya su interés en los procesos de construcción de contenidos y la significación que les otorgaban las audiencias, no se había desarrollado una línea específica sobre género y recepción.

Las quejas de las audiencias femeninas sobre la imagen generada por los medios han provocado un cierto interés de las instituciones públicas cuyas acciones se condensan en resultados cuantitativos sobre las opiniones y valoraciones de las audiencias. Las quejas de las audiencias sobre la representación estereotipada fueron motivo de algunas investigaciones como las de Courtney y Whipple (1983), Barry (et al. 1982). No obstante, comprender el significado que las audiencias otorgan a la representación de género en los medios de comunicación implica partir del papel activo de las personas receptoras. Una vez superada la idea de que los medios funcionan como agujas hipodérmicas que inyectan significaciones, representaciones e imaginarios y que en este proceso las audiencias solo participan como simples vasos contenedores, nos situamos en un momento en el que las personas consumidoras forman parte del proceso de significación (Martín Barbero, 1987; Orozco, 1987; Canclini, 2002).

Las audiencias activas pueden transformar el sentido de la recepción; en ella la identificación, como parte importante del consumo, no aparece como un "perder identidad por un convertirse en otro ajeno" (es decir, la alienación como pérdida de identidad, individual o colectiva), sino, por el contrario, como un "apropiarse de lo ajeno y nuevo, reconocido por alguna analogía con uno mismo" (Fuenzalida, Hermosilla, 1991: 30). Los estudios culturales ingleses nos ayudan a explicar cómo la audiencia es un agente social activo. Para estos estudios, "las audiencias no son 'zombies' culturales, sino activas 
productoras de sentido desde dentro de sus propios contextos culturales" (Ang 1996:185). El contexto en el que se lleva a cabo el consumo de los productos mediáticos y la vida cotidiana de los sujetos son dos elementos importantes para comprender el proceso de significación. De ese modo, "las audiencias pueden distinguir fácilmente entre ficción y realidad" (ibidem: 187).

El elemento que media el discurso que considera al sujeto con el poder suficiente para elegir y producir significados recae directamente en el matiz de los mensajes. Es decir, los contenidos mediáticos priorizan y proponen significaciones,

aunque el mensaje no sea un objeto con significado real, lleva en sí mecanismos significadores que estimulan ciertos significados, o incluso un significado privilegiado y, suprimen los demás: se trata de las "conclusiones directrices" codificadas en los mensajes (Morley 1998: 422).

La audiencia es el elemento clave de esta investigación, pero no hay que olvidar que nuestro objetivo es comprender cómo las mujeres perciben su imagen en los medios de comunicación. En definitiva, lo que proponemos es un estudio sobre la recepción de la imagen de las mujeres desde la perspectiva de género. Tomando en cuenta que nuestro modelo está alimentado a partir de los estudios culturales, entendemos que la representación es un proceso eminentemente social. Es una forma de entender "cómo el mundo está socialmente construido y representado para nosotros y por nosotros mediante la significación (Baker 2003: 37).

La investigación sobre género y comunicación ha estado vertebrada principalmente a partir de dos grandes líneas que han sido, por un lado, la imagen de las mujeres en las industrias culturales y, por el otro, su participación en los procesos de producción y decisión de los medios de comunicación. El estudio de recepción de esos discursos a partir de la perspectiva de género no es precisamente la línea que reúne un mayor número de investigaciones desarrolladas en el ámbito de las ciencias de la comunicación. Es por tanto necesario que las investigaciones sobre comunicación y género profundicen en los estudios de recepción de la representación de género en los medios. Dicho conocimiento permitirá matizar, explicar y completar el ciclo en el proceso comunicativo. 


\section{METODOLOGÍA}

Para indagar sobre la imagen femenina en los medios de comunicación utilizamos la técnica cualitativa de los grupos de discusión. Hemos optado por el conocimiento cualitativo porque nuestro objetivo esencial es aproximarnos a una realidad, entender las motivaciones del comportamiento social y las actitudes, en este caso, de las consumidoras en relación con la representación que los medios hacen de las mujeres. En cualquier caso, nos interesa analizar el discurso colectivo dirigido y conducido que se genera en los grupos de discusión.

Los grupos de discusión nos permiten describir y exponer determinados fenómenos sociales presentes en la realidad cotidiana de los sujetos. Este conocimiento es necesario para realizar una cartografía que nos permita establecer las coordenadas necesarias para mejorar realidades sociales concretas.

La muestra del estudio está constituida por 18 mujeres que fueron organizadas equitativamente en dos grupos de discusión. Las personas participantes en esta investigación se seleccionaron de un total de 34 mujeres que respondían a las siguientes variables: mujeres de edades comprendidas entre los 24 y los 45 años, con estudios secundarios o superiores, residentes en municipios de la provincia de Barcelona, con ocupación laboral en diversos sectores económicos, que formaran parte de familias integradas por dos o más miembros, y que hayan realizado o no alguna reclamación formal de diversa temática sociocultural.

Siguiendo con las pautas que rigen los grupos de discusión, consideramos que nueve sujetos para cada grupo de discusión eran suficientes para lograr los objetivos marcados. La variable diferencial entre los grupos era que uno estaba integrado por mujeres que habían realizado alguna reinvindicación formal de tipo sociocultural y otro integrado por mujeres que nunca había formulado explícitamente una queja también de carácter sociocultural. De tal forma, uno de los grupos estaba constituido por mujeres más activas socialmente y el otro por personas con poca capacidad de agencia en su entorno social.

Para cada grupo de discusión se contempló una duración de dos horas aproximadamente. Las sesiones, se realizaron en la sala de trabajo 
del Grup de Investigació en Imatge So i Síntesi (GRISS) de la Universidad Autónoma de Barcelona.

Previo al inicio de la discusión, los sujetos visionaron tres productos audiovisuales difundidos en diferentes medios de comunicación. Estos productos visionados en los grupos de discusión fueron seleccionados entre los contenidos de los medios de comunicación denunciados o felicitados por la audiencia en Cataluña. En dichos productos están presentes representaciones que mostraban a mujeres estereotipadas a partir de concepciones sexistas y propuestas no discriminatorias, en las que el hombre y la mujer no aparecen relacionados con estereotipos convencionales o sexistas. Los anuncios visionados en la sesión correspondían a publicidad que había sido analizada y valorada en el servicio de quejas y prácticas positivas que ofrece el Institut Català de les Dones de la Generalitat de Catalunya. A través de este servicio la ciudadanía puede enviar sus quejas o felicitaciones sobre productos de los medios de comunicación en relación con los contenidos sexistas de los medios (http://www.gencat.net/icdona).

La dinámica de los grupos estaba regida por una serie de categorías temáticas, entre las que destacan: estereotipos convencionales y no convencionales en la representación de las mujeres en los medios de comunicación, relación y ambigüedad entre representación y la realidad social, discursos sociopersonales sobre la imagen de la mujer en el ámbito privado (relaciones sociales, personales y profesionales, prospectivas sobre el tratamiento de las mujeres en los medios y otros soportes de contenidos multimedia, y conocimiento sobre las instituciones públicas que tienen como objetivo mejorar la situación de las mujeres en diversos ámbitos sociales y culturales.

\section{RESULTADOS}

\section{Pautas para identificar y explicar}

la visibilización discriminatoria o no discriminatoria

Para las mujeres de los grupos de discusión, la representación del género femenino en los medios de comunicación se presenta generalmente con patrones que infravaloran la imagen de su propio género. Los clichés principales destacados por las mujeres de los dos grupos corres- 
ponden a la invisibilización y ridiculización de la mujer, a la permanencia del rol de la mujer como víctima, a la coexistencia de estereotipos entre la mujer y la belleza, a la perpetuación de roles tradicionales que asocian a las mujeres con un ámbito privado-doméstico, a la cosmovisión masculina como dinámica arquetípica a seguir por las mujeres, a la presencia de la mujer-objeto en los medios y, finalmente, a la especialización temática de las revistas en función de los géneros.

Para las mujeres de los grupos, el imaginario que plantean los contenidos mediáticos, específicamente el que propone la publicidad, se construye a partir del menosprecio y la tipificación de los roles de género. $\mathrm{Al}$ respecto una mujer señalaba: "La publicidad antes ridiculizaba a las mujeres. Y ahora parece que las cosas han cambiado. Pero lo que hacen es ridiculizar también a los hombres". En este sentido, el predominio en la publicidad de un imaginario masculino y su reproducción como pauta a seguir dentro del ámbito femenino se valora negativamente: "Siempre imitando al hombre. Nunca como mujer misma. Pero en realidad no es que hagas lo mismo sino que siendo mujer te valoren". "Yo creo que hemos pasado de representar una señora que discute con las amigas en el supermercado sobre qué detergente es el mejor, a ser mujeres súper agresivas con su cartera y trabajando sin parar".

Uno de los criterios de noticiabilidad que aumenta la discriminación de los medios en relación con las mujeres, según las integrantes de los grupos, es su presencia como víctima de la violencia de género. El déficit generado en la representación femenina por este fenómeno, en concreto, se resume en dos aspectos: el primero, es la constatación del protagonismo de las mujeres en los informativos cuando han sido víctimas de agresiones. El segundo aspecto se refiere al tratamiento informativo de las noticias con respecto a estos hechos. Existe un exceso de sensacionalismo en el tratamiento relativo a las mujeres y una clara ausencia del seguimiento de los casos y las consecuencias de las acciones de los agresores. Al respecto, una mujer explicaba en una de las sesiones:

"Las mujeres aparecen en los medios de comunicación como víctimas o como objetos. ¿Donde está nuestra capacidad de ser agencia? Las mujeres existimos como víctimas de hechos violentos, pero hay un trasfondo porque nada más existimos cuando se cierran prostíbulos...”. 
Algunas de las preocupaciones expresadas en el grupo son la difusión de estereotipos sobre las mujeres y su relación, casi exclusiva, con ámbitos privados (como responsables de las tareas domésticas y familiares) y el tratamiento que se hace de ellos en la publicidad. "Si ya pasas de los 20 años y no te ves estupenda, pues pasas al estereotipo de ama de casa, madre que compra detergente y pasa la aspiradora".

Uno de los arquetipos mencionado en las sesiones es la relación de las mujeres con el cuidado de la imagen y el concepto comercial de la belleza. Ambas cuestiones son percibidas como valores presentes en los medios que menosprecian la representación del género femenino en el ámbito social. Una mujer del grupo de consumidoras que no ha realizado alguna queja formal comentaba: "Ayer en un suplemento había un artículo de belleza que decía que tipo de maquillaje debes ponerte para estar bronceada todo el año. ¿Pero, es necesario estar bronceada?” Otra mujer indicaba: “¡No entiendo por qué no salen casas normales y gente normal!”. La publicidad dirigida a las que trata de identificarlas con un único canon de belleza se valora negativamente por el conjunto de la muestra. Los esteretipos que dibujan a las mujeres a partir de un modelo de belleza para conseguir el éxito con los hombres también están presentes en la discusión de los grupos. Las mujeres manifiestan que un sector de la población que es susceptible a este tipo de mensajes es el juvenil, principalmente las adolescentes. Puntualizan que en las revistas se reproducen discursos que vinculan a la mujer con el hombre, reduciendo el escenario de la mujer a un ámbito afectivo. "El mensaje que yo creo que están dando estas revistas para las adolescentes es que el tema principal y básico es su vida con el hombre. Y que su identidad se basa en si puede ligar mucho o no".

Un modelo sexista presente en la política editorial de las revistas es la especialización temática que responde a cánones estereotipados de género: ofrecen prensa del corazón para las mujeres, y política, economía o deporte para los hombres.

"Mis referentes son las revistas. Hay una clara diferencia entre las revistas femeninas y las revistas masculinas. ¡Y es muy bestia! Las femeninas engloban todo el tema de atención física, productos de belleza en general, revistas del corazón, tendencias... Me gusta la decoración y la cocina, pero un artículo de política en su momento también va bien”. 
Las ausencias sobre la imagen de las mujeres

La invisibilización de las mujeres, en diferentes ámbitos, se aprecia como un déficit respecto a una construcción equilibrada del universo femenino y respecto al masculino. Este déficit genera una contradicción entre la representación, que construyen los medios mediante sus contenidos en materia de género y la realidad social.

La referencia a la abstracción del contexto femenino se concreta en los siguientes aspectos:

1. La sexualización de los planteamientos editoriales de las publicaciones periódicas, lo que provoca una ausencia de temáticas de interés para las mujeres.

2. Invisibilización de las mujeres en los informativos.

3. La presencia de la imagen de las mujeres como víctimas en los contenidos informativos de los medios.

3. Escasa presencia de mujeres en la producción de contenidos.

4. Ausencia de una representación de las mujeres que no sea maniqueísta desde la perspectiva de género.

5. Ausencia de la representación de la mujer a partir de su propia cotidianidad.

La ubicación socioespacial de las mujeres es un aspecto importante que se observa en este estudio, principalmente sobre aquellos espacios donde las mujeres no figuran. Se trata de las áreas que las propias consumidoras de los mensajes valoran como fundamentales en función de la realidad de cada una de las personas.

Las integrantes de ambos grupos han destacado dos características que generan plusvalía en los estereotipos utilizados en los contenidos mediáticos, principalmente en los discursos publicitarios. La primera, la introducción del tema de la conciliación y/o corresponsabilidad familiar y, la segunda, la difusión de patrones femeninos que no responden a los arquetipos tradicionales de belleza.

Las receptoras valoran positivamente la inserción del tema de la conciliación laboral en la agenda periodística, así como su tratamiento en determinadas campañas publicitarias. Una mujer argumentó: "Yo recuerdo muy bien un anuncio donde se veía una mujer que iba vestida 
como una ejecutiva con un carro de la compra, el bebé, la cartera... ¡Y el marido sin nada! ¡Sin bebé! Estaba bien”. La equidad en la responsabilidad familiar entre hombres y mujeres que se representan en las campañas publicitarias se asume como una propuesta constructiva de la realidad social. A este criterio, se suman los anuncios que muestran mujeres con atributos físicos distintos a los dictados por los arquetipos de belleza. Las mujeres valoran positivamente los contenidos que proponen otras representaciones del cuerpo femenino. "Está bien lo de "Mujeres reales" [referencia a una campaña de productos para el cuidado personal], que salgan modelos que no salen normalmente. Pues se agradece bastante", explicaba una de los sujetos de la muestra.

Siguiendo con lo expresado por las participantes en la discusión, algunas de ellas observan que la política editorial se ha adaptado al contexto social, sobre todo en lo que concierne a cuestiones de género. Lo anterior se concreta en el rol protagonista de las mujeres en diversos ámbitos como el social, el político o el cultural.

"En muchas ocasiones, ahora más que antes, las portadas se hacen para las amas de casa o para las mujeres que están en el poder. Así pienso que en algunos medios ha habido un cambio de línea para dar otra imagen a las mujeres".

Los elementos significativos que generan un déficit en la imagen de las mujeres expresan contradicciones entre la realidad social y la mediática. El estudio de estos elementos explica precisamente esta disociación.

\section{Estrategias para la representación no sexista}

Durante el desarrollo de las sesiones, las mujeres fueron cuestionadas sobre las posibles vías de acción para tratar de corregir los estereotipos sexistas en los medios de comunicación. Al respecto, destacaron algunas propuestas que se ubican en diferentes escenarios:

1. Relación directa entre opción de compra de los productos a partir de sus campañas publicitarias. ("Si yo veo el anuncio de... y no me gusta. Me quedo con la marca". "Si a mí aquella mujer no me gusta, no compraré el producto"). 
2. Trabajar cuestiones de género desde el ámbito de la educación formal y no reglada. ("Yo creo que la clave es la educación, que no deberían llegar a esta situación de saturación para decidirnos").

3. Facilitar, agilizar y simplificar la información y tramitación de quejas o reconocimientos a empresas, organizaciones y/o instituciones que generen discursos de género. ("Hay una cosa del derecho al consumo, creo").

4. Estimular la acción de participación social contra la representación de las mujeres desde la reflexión y la crítica. ("Yo pienso que la responsabilidad es de todas las mujeres que se sientan ofendidas cuando ven un anuncio. Te indignas, pero también piensas... ya se lo harán”).

5. Impulsar representaciones diversas sobre las mujeres en los medios. ("Sería interesante ofrecer nuevos modelos de televisión, tanto como mujeres hay en la vida cotidiana").

6. Potenciar organismos $y / o$ instituciones que regulen y controlen la imagen de las mujeres en los medios. ("No puedes estar todo el día analizando. No sé...yo creo que debería de haber un organismo de control que se dedique a eso").

\section{APROXIMACIÓN AL PERFIL DE RECEPCIÓN PASIVA Y RECEPCIÓN ACTIVA DE LAS MUJERES}

Este estudio sobre la recepción de los contenidos de los medios no observa grandes divergencias de consumo entre las mujeres más o menos activas con relación a sus manifestaciones sobre la imagen de las mujeres en los medios. En ambos grupos surge la constatación de los estereotipos que infravaloran la imagen de la mujer y la percepción favorable hacia aquellos contenidos que generan plusvalía.

Este fenómeno implica que los estereotipos se consumen a partir de un análisis crítico del discurso en diferentes niveles. La disociación entre realidad y contenidos mediáticos se identifica y argumenta como un hecho de facto en nuestra sociedad que comporta la pérdida de la función constructiva de los medios de comunicación. Este hecho pone de manifiesto la necesidad de valorar si el consumo de las diversas representaciones, propuestas por los medios, puede estar determinado por variables de carácter sociocultural o demográfico de las audiencias. El 
estudio presentado trabaja una línea de investigación sobre la presencia de significaciones mediáticas que perpetúan arquetipos tradicionales de género y que son percibidas por las mujeres receptoras en su conjunto.

El análisis de las posibles convergencias y divergencias entre las participantes de ambos grupos determina dos ejes temáticos sobre los que se han generado los resultados expuestos. El primer eje describe e interpreta los elementos que generan contenidos sexistas al margen de la propia realidad cotidiana, y el segundo eje expone las acciones y vías para mejorar esta realidad. Sobre el primero no se observan divergencias entre los diversos grupos de receptores. Sin embargo, en el segundo eje encontramos algunas diferencias cualitativas importantes. Entre estas destacan la aparente actitud conformista acompañada de ciertas perspectivas fatalistas, la delegación de las funciones reivindicativas o de denuncia en las asociaciones que trabajan desde la perspectiva de género, y una falta de información o desconocimiento sobre la posibilidad de hacer una denuncia o reclamación. La manifestación de actitudes conformistas, por parte del grupo de receptoras menos activas, es una constante en su discurso; sin embargo, este comportamiento va acompañado de indignación sobre la representación mediática de las mujeres. Los contenidos mediáticos que contienen representaciones estereotipadas del género femenino les molestan, se observa una reacción, pero no logran concretar este sentimiento en una acción que las involucre como agentes de cambio de la realidad social. Una mujer opinaba: "Puede llegar a todos y puede ser que a nosotros no nos agrada, pero es lo que hay".

El conformismo observado se conceptualiza en una actitud social que establece una jerarquía entre problemas sociales de primero y segundo grado. "No nos quejamos porque las hipotecas están altísimas. Entonces tampoco nos quejaremos por un anuncio porque tampoco es tan trascendente." De este modo, según esta jerarquía, la representación de la mujer pasa a un segundo plano de importancia.

El segundo elemento que plantea diferencias entre ambos grupos es la delegación en las asociaciones que trabajan con temas de discriminación femenina, cuando se trata de promover acciones reivindicativas.

El desconocimiento o falta de información difundida desde las instituciones catalanas encargadas de la defensa de un imaginario femenino equilibrado es una de las cuestiones que nos ayudan a comprender 
la pasividad de uno de los grupos. Este desconocimiento está presente incluso entre las mujeres más activas, quienes manifiestan una actitud participativa en cuestiones de género. La única asociación referida durante las sesiones ha sido la Organización de Consumidores y Usuarios (OCU), que destaca la complicación y la dificultad de las tramitaciones personales. Al respecto, una mujer opinó:

"Tampoco vas y dices: bien, voy a hacer una queja a la OCU. Porque muchas veces pienses que no servirá de nada el hecho que estés en contra... Te deberían de facilitar las cosas (...) Ahora has de agarrar el teléfono, has de llamar a la OCU, te pasan con atención al cliente... Son más las cosas que has de dar, llenar las instancias, después ir a plaza Cataluña, y no sé donde más (...) No te dan facilidades. ¡Si fuese una cosa más fácil!”

Otra mujer lo concretaba así: “iSi fuese [tan fácil] como llamar a Gran Hermano!". Finalmente, y al hilo de lo expresado, las consumidoras demandan mayor información para acceder a los mecanismos institucionales que les permitan participar como agentes sociales y tratar de coadyuvar al cambio en cuestiones de género.

\section{CONCLUSIONES}

Los trabajos de investigación realizados sobre los estereotipos de las mujeres parten de la complementariedad de los análisis desarrollados desde la producción, desde el análisis del discurso textual de las producciones audiovisuales y desde los estudios sobre la recepción. Tanto los estudios sobre la cultura desarrollados en Latinoamérica como los estudios culturales (ingleses y/o norteamericanos) son piezas clave para entender y abordar la recepción de la imagen de las mujeres en los medios, así como las aportaciones de las teorías feministas y desde la perspectiva de género de las últimas cuatro décadas. Es precisamente la complementariedad teórica y multidisciplinar la que permite avanzar en el conocimiento generado para evitar la discriminación sexista de los contenidos expuestos en las industrias culturales.

La transversalidad de la variable género en las investigaciones de ámbito social, humanístico y técnico no ha estado siempre presente. 
Por otro lado, la investigación sobre género y comunicación, y/o mujer y comunicación, se constituye como una línea de investigación de la disciplina de las ciencias de la comunicación. Posiblemente, la falta de recursos definidos y garantizados por las políticas científicas de las diversas instituciones gubernamentales ha sido uno de los elementos importantes en la valoración de las tradiciones y otras trayectorias históricas en temas de investigación de las distintas comunidades.

Sabemos que para profundizar en el estudio de las industrias culturales, específicamente en los estudios de los medios de comunicación, necesitamos conocer y valorar la producción a partir de los contenidos y desde una perspectiva etnográfica del estudio de su recepción. Y no ha sido esta última una de las más incentivadas desde los estudios de comunicación. Los trabajos desarrollados desde las ciencias de la comunicación en materia de estereotipos y medios no siempre han tenido una aproximación cualitativa a partir del discurso de las audiencias femeninas.

El relato etnográfico de la audiencia, y concretamente de las receptoras de los discursos narrativos de los medios de comunicación, es una pieza clave para entender determinadas realidades. Los resultados de este estudio pretenden incidir en la importancia de las percepciones y consideraciones de la ciudadanía frente a los contenidos que generan los medios, pero también en la explicación del comportamiento de las audiencias en relación con la participación social referente a los contenidos de los medios, y la valoración en la estrategia, efectividad y otras acciones que permitan mejorar los contenidos producidos en la industria audiovisual.

\section{Bibliografía}

ANG, I. (1996) Living room wars. rethinking media audiences for a postmodern world, Routledge.

BAKER, C. (2003) Television, globalización e identidades culturales, Barcelona: Paidós.

BELKAOUI, A. y Belkaoui, J. (1980) "A comparative analysis of the roles portrayed by women in print advertisements: 1958, 1970, 1972", Journal of Marketing Research, vol. 13, Chicago: American Marketing Association. 
GARCÍA CANCLINI, N. (2002) Culturas populares en el capitalismo, México: Grijalbo.

CEulemans, M., Fauconnier, G. (1980) Imagen, papel y condición de la mujer en los medios de comunicación social, UNESCO/Études et documents d'information, núm. 84, París.

COURTNEY A. y Whipple T. (1983) Sex stereotyping in advertising, Toronto: Lexington Books.

CURRAN, J. et al. (ed.) (1998) Estudios culturales y comunicación. Análisis, producción y consumo cultural de las políticas de identidad y el posmodernismo, Barcelona: Paidós.

FERGUSON, J. Et al. (1990) "In the pages of ms: sex role portrayals of women in advertising", Journal of Advertising, vol. 19.

FRANQUET, R. (1992) "Mujeres y rutinas de producción en radio y televisión" en La investigación en la comunicación. III Simposio de la Asociación de Investigadores en Comunicación, Madrid: ICE.

FUENZALIDA, V., Hermosilla, M. (1991) El televidente activo. Manual para la recepción activa de TV. Santiago de Chile: s/e.

FURNHAM, A., Babitzkow, M., Uguccioni, S. (2000) "Gender stereotyping in television advertisements: a study of french and danish television", Genetic, Social, and General Psychology Monographs, 126 (1), (febrero, 2000).

GALlaGHER, M. (1981) Unequal opportunities. The case of women and the media. París: Unesco.

GALLEGO, J. (2002) "La prensa diaria por dentro: mecanismos de transmisión de estereotipos de género en la prensa de información general", Anàlisi 28.

GOFFMAN, Erving. (1976) Gender advertisements. Londres: McMillan Press.

GREIMAS, A. (1973) En torno al sentido: ensayos semióticos, Fragua.

HALL, J. (1994) Mighty minutes: an illustrated history of television's best commercials, New York: Harmony Books.

KLASSEN, M. L. et al. (1993) "Men and women: images of their relationships in magazine advertisements" Journal of Advertising Research, vol. 23, núm 2. UK: World Advertising Research Center Limited. 
KIVIKURU, U. (1999) “Images de la femme dans les médias", en $R a-$ pport sur les recherches dans l'Union Européenne, Emploi \& Affaires Sociales, Bruselas, Comission Européenne.

KovACS, M. (1972) "Women simply don't recognize themselves in many ads today", Advertising Age, vol. 12 (6).

LULL, J. et al. (1977) "Recognition of female stereotypes in TV commercials", Journalism Quarterly, vol. 54, 1, Spring 1977.

LUNDSTROM, W., Sciglimpaglia, D. (1977) "Sex role portrayals in advertising", Journal of Marketing, núm. 41.

LYSONSKI, S. (1985) "Role portrayals in british advertisements", European Journal of Marketing, vol. XIX, núm. 7.

FUENZALIDA, V., Hermosilla, M. (1991) El televidente activo. Manual para la recepción activa de TV, Santiago de Chile: s/e.

MANSTEAD, A. y McCULLOCH, C. (1981) "Sex-role stereotyping in british television advertisements", British Journal of Social Psychology, 20, 1981.

MARTÍN-BARBERO, J. (1987) De los medios a las mediaciones, Barcelona: Gustavo Gili.

MASSE, M.. A.; Rosenblum, K. (1988) "Male and female created they them: the depiction of gender in the advertising of traditional men's and women's magazines", Women's Studies International Forum, vol. 11, núm. 2. UK: Elsevier.

MCROBBIE, A. (1998) "More!: nuevas sexualidades en las revistas para chicas y mujeres" en Curran, James, M., David y Walkerdine, V. (eds.) Estudios culturales y comunicación, Barcelona: Paidós.

MORLEY, D. (1988) "Populismo y revisionismo y los 'nuevos' estudios de audiencia“, en Curran, Morley, Walkerdine, Estudios culturales y comunicación. Análisis, producción y consumo cultural de las políticas de identidad y el posmodernismo, Paidós: Barcelona.

O'DONELL, W. O'donell, K. (1978) 'Update: sex-role messages in tv commercials", Journal of Communication, núm.1, vol. 28, 1978.

OROZCO, G. (1987) "Televisión y producción de significados", en Revista Comunicación y Sociedad, Cuadernos del CEIC, Difusión científica.

PINGREE, S., et al. (1976) “A scale of sexism”, Journal of Communication, vol. XXIII, núm. 4, 1976. 
RuBlE, D. N., y Ruble, T. L. (1982) "Sex stereotypes", en Miller In the eye of the beholder. Contemporary issues in stereotyping, Nueva York: Praeger.

SEXTON, D., Haberman, P. (1974) "Women in magazine advertisements", Journal of Advertising Research, vol. XIV, núm. 4, 1974.

VAN ZOONEN, L. (1995) Gender representation and the media”, en Downing et al. (eds.) Questioning the media, Londres: Sage.

WoOD, J. (1994) Gendered lives: communication, gender and culture, California: Wadsworth.

WOODWARD, K. (1997) Identity and difference, Londres: Sage.

ZOOMEN, L. (1994) Feminist media studies, Londres: Sage. 